\title{
Efeitos da imersão nos parâmetros ventilatórios de pacientes com distrofia muscular de Duchenne
}

\section{Effects of immersion on the breathing parameters of patients with Duchenne muscular dystrophy}

Camila de Almeida ${ }^{1}$, Raphael Augusto Fernandes de Oliveira ${ }^{2}$, Daiane Spalvieri ${ }^{3}$, Douglas Braga ${ }^{4}$, Maria Misao ${ }^{4}$

\section{RESUMO}

Em muitos centros de reabilitação, a fisioterapia aquática é utilizada para o tratamento de pacientes com distrofia muscular de Duchenne (DMD). Entretanto, são escassas as evidências científicas sobre os efeitos da imersão nos parâmetros ventilatórios desses pacientes. Objetivo: Avaliar os efeitos da imersão no nível da sétima vértebra cervical (C7) com relação aos parâmetros ventilatórios de pacientes com DMD. Método: Participaram do estudo quinze meninos com diagnóstico de DMD e média de idade de 12 anos. Coletados dados gerais e história clínica, os pacientes foram avaliados em solo e em imersão sob seguintes parâmetros: Saturação parcial de oxigênio $\left(\mathrm{SpO}_{2}\right)$, frequência cardíaca (FC), pressões inspiratórias (PI Máx.) e expiratórias (PE Máx.) máximas, Volume Minuto (VM), Frequência Respiratória (FR), Volume Corrente (VC), Capacidade Vital Forçada (CVF) e Pico de Fluxo Expiratório (PFE). A SpO 2 sofreu uma redução após a avaliação no meio líquido quando comparada aos valores anteriores à avaliação no mesmo meio $(p=0,01)$. Resultados: A FR foi maior em meio líquido que em solo $(p=0,02)$. As PI Máx. e as PE Máx. não se modificaram em meio líquido. Dentre os volumes analisados, a CVF e o PFE apresentaram valores menores em meio líquido quando comparada aos valores mensurados em solo $(p=0,004)$. VM e VC não sofreram alteração. Ao se relacionar os valores de CVF em solo e em meio líquido, assim como, os valores de PFE, observa-se que há correlação positiva entre os dados coletados em solo e em meio líquido (CVF: $r=0,692$, $p=0,006$; PFE: $r=0,913, p=0,0001$ ). A imersão no nível de C7 foi capaz de reduzir a CVF e o PFE de pacientes com DMD, bem como provocar aumento da FR dos mesmos. Conclusão: Com os dados de correlação entre valores em solo e em meio líquido da CVF e do PFE, há possibilidade de se sugerir um valor para estas variáveis em meio líquido a partir de valores mensurados em solo. Estes dados podem fornecer um melhor embasamento para avaliar a indicação de atividades em meio líquido para pacientes com DMD, em diferentes estágios de evolução da doença.

Palavras-chave: distrofia muscular de duchenne, hidroterapia, imersão, ventilação pulmonar

${ }^{1}$ Fisioterapeuta, Instituto de Reabilitação Lucy Montoro.

${ }^{2}$ Fisioterapeuta, pela Universidade de São Paulo (USP).

${ }^{3}$ Fisioterapeuta, Docente na Universidade Nove de Julho (UNINOVE).

${ }^{4}$ Fisioterapeuta, Associação de Assistência a Criança Deficiente (AACD).

Endereço para correspondência: Instituto de Reabilitação Lucy Montoro - Unidade Ribeirão Preto

Camila de Almeida

E-mail: camilalmeidaft@hotmail.com

Recebido em 25 de Abril de 2011.

Aceito em 02 de Fevereiro de 2012.

Pesquisa realizada na Associação de Assistência à Criança com Deficiência (AACD).

DOI: $10.5935 / 0104-7795.20120005$

\begin{abstract}
In many rehabilitation centers, Aquatic Physiotherapy is commonly used as an optional treatment for Duchenne Muscular dystrophy (DMD) patients. However, there is so little scientific evidence about the immersion effects on the breathing parameters of these patients. Objective: Our goal was to evaluate the immersion effects to the depth of the seventh cervical vertebra (C7), related to the breathing parameters in DMD patients. Method: Fifteen boys with DMD participated of the study, averaging 12 years in age. Clinical history and general information were investigated, and the following parameters were evaluated on the ground and inside the pool: Partial Oxygen Saturation $\left(\mathrm{SpO}_{2}\right)$, heart rate ( $\left.\mathrm{HR}\right)$, maximal inspiratory (PI Max.) and expiratory (PE Max.) pressures, minute volume (MV), respiratory rate (RR), tidal volume (TV), Forced Vital Capacity (FVC) and peak expiratory flow (PEF). The $\mathrm{SpO}_{2}$ diminished after the aquatic evaluation when compared to values beforehand $(p=0.01)$. RR was higher inside the pool than outside $(p=0.02)$. PI Max. and PE Max. did not change inside the pool. Results: Analyzing the results for volumes, FVC and EPF were reduced inside the pool when compared to evaluation on solid ground $(p=0.004)$. MV and TV did not change. A positive correlation between ground and pool values of FVC and PEF was seen (FVC: $r=0.692, p=0.006$; EPF: $r=0.913, p=0.0001$ ). $C 7$ immersion was able to reduce $\mathrm{SpO}_{2}, \mathrm{FVC}$, and PEF, while increasing the RR of DMD patients. Conclusion: With the correlation between ground and pool values of FVC and PEF encountered in this study, there is a possibility of predicting pool values for these parameters using only the ground data. These findings could lead to a scientific base for a better Aquatic Physiotherapy prescription for DMD patients, at different pathology stages.
\end{abstract}

Keywords: hydrotherapy, immersion, muscular dystrophy duchenne, pulmonary ventilation 


\section{INTRODUÇÃO}

Distrofia Muscular de Duchenne (DMD) é uma doença caracterizada por perda progressiva de força muscular, contraturas, atrofias e deformidades, resultando em perda da marcha, comprometimento dos músculos respiratórios e morte por insuficiência respiratória. A incidência é de aproximadamente 1:3000 meninos nascidos vivos. ${ }^{1,2}$ Há um importante avanço nos cuidados respiratórios destes pacientes devido ao alto índice de mortalidade relacionado com a alteração no sistema pulmonar. ${ }^{2}$

A capacidade vital forçada (CVF) é um importante parâmetro respiratório que pode refletir o estado clínico do indivíduo. Em doenças neuromusculares progressivas, por volta da idade em que a deambulação é perdida, a CV tende a cair progressivamente..$^{3-12}$

A fisioterapia aquática busca otimizar a função muscular global, inclusive dos músculos associados à respiração, e facilitar a movimentação ativa através das propriedades do meio líquido. No entanto, o sistema pulmonar é amplamente afetado pela imersão. ${ }^{13}$

Quando indivíduos saudáveis são imersos no nível do pescoço, o trabalho respiratório total, para um volume corrente de $1 \mathrm{~L}$, aumenta em torno de $60 \%$. Em decorrência deste esforço, três quartos são atribuídos ao aumento do trabalho elástico (redistribuição do sangue da periferia para o tórax) e o restante em decorrência do trabalho dinâmico (movimento do ar contra uma resistência aérea aumentada e aumento da pressão hidrostática no tórax). ${ }^{14-17} \mathrm{O}$ efeito combinado destes fatores altera a função pulmonar, aumenta o trabalho respiratório e muda a dinâmica respiratória. ${ }^{13}$

A maioria da literatura disponível é oriunda de estudos fisiológicos e aeroespaciais caracterizada por pouca evidência baseada em âmbito clínico. Além disso, a literatura baseia-se em indivíduos saudáveis para descrever os efeitos da imersão no sistema pulmonar. ${ }^{13,18,19}$

\section{OBJETIVO}

O objetivo deste trabalho é avaliar os efeitos da imersão nos parâmetros ventilatórios em pacientes com DMD no nível da sétima vértebra cervical.

\section{MÉTODO}

Este estudo de caráter transversal foi realizado na Associação de Assistência à Criança Deficiente (AACD) e seu projeto foi aprovado pelo Comitê de Ética da mesma Instituição sob o parecer n030/2008. A execução está de acordo com a resolução $n^{\circ} 01 / 88$ do Conselho Nacional de Saúde.

Foram selecionados pacientes com diagnóstico de distrofia muscular de Duchenne, acompanhados regularmente na AACD. Todos preencheram os critérios de inclusão do protocolo proposto: diagnóstico de DMD estabelecido por meios de achados clínicos e confirmado por biópsia muscular; controle de tronco em superfície estável; grau de colaboração compatível com a realização da avaliação respiratória; obtenção do Termo de Consentimento Livre e Esclarecido (TCLE) e ausência de episódios de pneumonia no mês antecedente ao da avaliação.

As avaliações foram realizadas no setor de Hidroterapia da Instituição em dois momentos distintos, num intervalo de 7 dias, pelo mesmo examinador, previamente treinado. Em uma sessão, foi realizada avaliação em solo com o paciente sentado no tablado (altura de $42 \mathrm{~cm}$, comprimento de $2,0 \mathrm{~m}$ e largura de 1,40 m). Em outra sessão, foi realizada avaliação em meio líquido com o paciente sentado no tablado $(84 \mathrm{~cm}$ largura, $1,21 \mathrm{~m}$ de comprimento, $42 \mathrm{~cm}$ de altura) imerso no nível da sétima vértebra cervical, em uma piscina terapêutica (largura de 3,5 $\mathrm{cm}$, comprimento de $12 \mathrm{~m}$, profundidade de $1 \mathrm{~m}$ e temperatura de $33,5^{\circ} \mathrm{C}$ ). A ordem de realização das avaliações foi escolhida de forma aleatória.

Todos os responsáveis pelos participantes foram esclarecidos quanto ao objetivo da pesquisa e, após a obtenção do TCLE, responderam a um breve questionário. Durante as avaliações, foram mensurados: Saturação parcial de oxigênio $\left(\mathrm{SpO}_{2}\right)$, Frequência cardíaca ( $\left.\mathrm{FC}\right)$, Volume minuto (VM), Frequência respiratória (FR), Volume corrente (VC), Capacidade vital forçada (CVF), Pressão inspiratória máxima (PI Máx.), Pressão expiratória máxima (PE Máx.) e Pico de fluxo expiratório (PFE). Realizadas três medidas de cada variável respiratória, considerou-se a de maior valor obtido.

$\mathrm{A} \mathrm{SpO}_{2}$ e $\mathrm{FC}$ foram mensuradas através do Oxímetro de pulso portátil de dedo Tipo Clip Onix 9500 Nonim $^{\circledR}$. Os volumes e as capacidades foram mensurados por meio da ventilometria, efetuada por um Ventilômetro Ferraris Respirometer, Wright $M K^{\circledR}$.
Com intuito de verificar de forma indireta a força muscular respiratória, as pressões respiratórias estáticas máximas (PI Máx e PE Máx) foram mensuradas através do manovacuômetro da marca Gerar classe $B^{\circledR}$, capaz de medir pressões negativas e positivas.

O pico de fluxo expiratório foi quantificado por meio de um medidor de fluxo expiratório Mini Wright ${ }^{\circledR}$.

Os dados de $\mathrm{SpO}_{2}$, FC, FR, VC, VM, PI Máx., PE Máx., CVF e PFE foram avaliados por análise não paramétrica de Wilcoxon. Para análise da CVF e PFE, as correlações de Spearman, seguidas de Regressão Linear, foram utilizadas para relacionar dados coletados em solo e em meio líquido.

\section{RESULTADOS}

A média de idade dos pacientes é de 12 anos, o peso, de 40,18 kg e a altura média, de 1,43 m. Dos 15 pacientes, 9 são cadeirantes. Os pacientes perderam a marcha, em média, há 3,38 anos. Nenhum dos pacientes faz uso de bi-level positive airway pressure.

Dos 15 pacientes, apenas um não realiza fisioterapia ou fisioterapia aquática. O restante realiza fisioterapia, fisioterapia aquática, natação ou yoga.

Nos dados coletados através do oxímetro observou-se que não houve diferença nas medidas de frequência cardíaca, entre o solo e o meio líquido $(p=0,951)$ e após a avaliação no meio líquido $(p=0,518)$. Nos dados de oximetria também não foi encontrado alteração entre os valores do solo e do meio líquido. Porém, os dados obtidos após a avaliação no meio líquido apresentaram-se menores quando comparados aos valores anteriores à avaliação neste meio ( $p=0,012$ ) (Figura 1$)$.

Nos dados de pressão coletados através do manovacuômetro, não houve diferença na PI Máx. $(p=0,240)$ e na PE Máx. ( $p=$ $0,946)$ dentro e fora do meio líquido.

Com relação aos dados volumétricos aquisitados pelo ventilômetro pudemos notar que o VM está no limite para se tornar estatisticamente maior no meio líquido quando comparado com a avaliação no solo $(p=0,058)$. O VC não apresentou diferença quando analisado dentro e fora da água $(p=0,391)$. Em contrapartida, a FR apresentou-se maior no meio líquido quando comparado com os valores de solo $(p=0,029)$ (Figura 2 ).

A CVF apresentou-se menor no meio líquido quando comparado com o solo $(p=0,008)$, e quando foram correlacionados os valores dentro e fora do meio líquido, foi observado uma correlação positiva entre eles $(r=0,692 ; p=0,006)$ (Figura 3). 
Meio Líquido

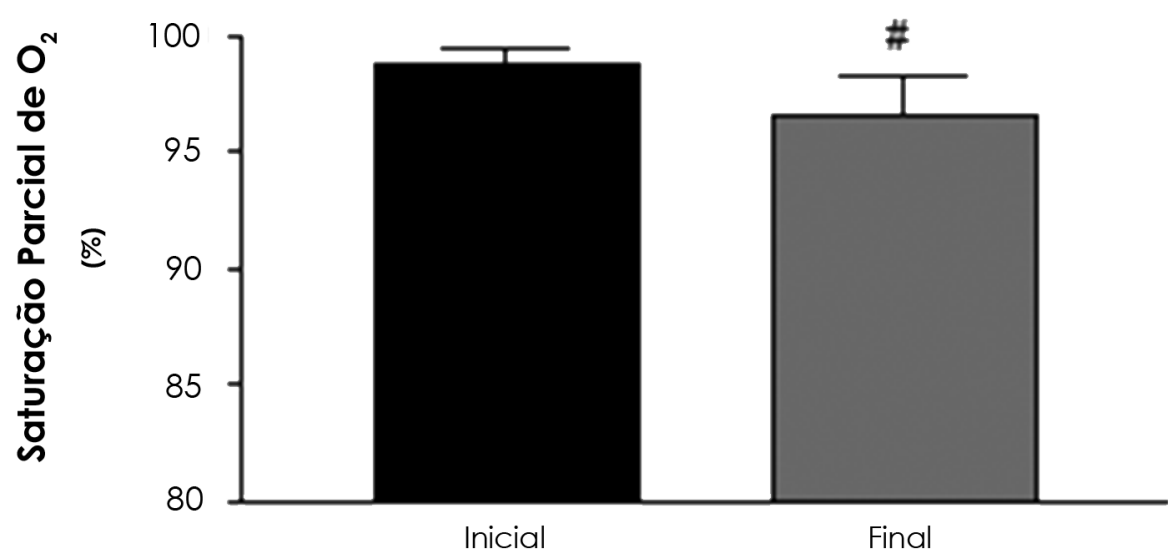

Figura 1. Dados de saturação parcial de oxigênio coletados em meio líquido em pacientes com DMD antes e após a avaliação. \# vs. Inicial (Wilcoxon, $p<0,05$ ); $\mathrm{N}: 15$
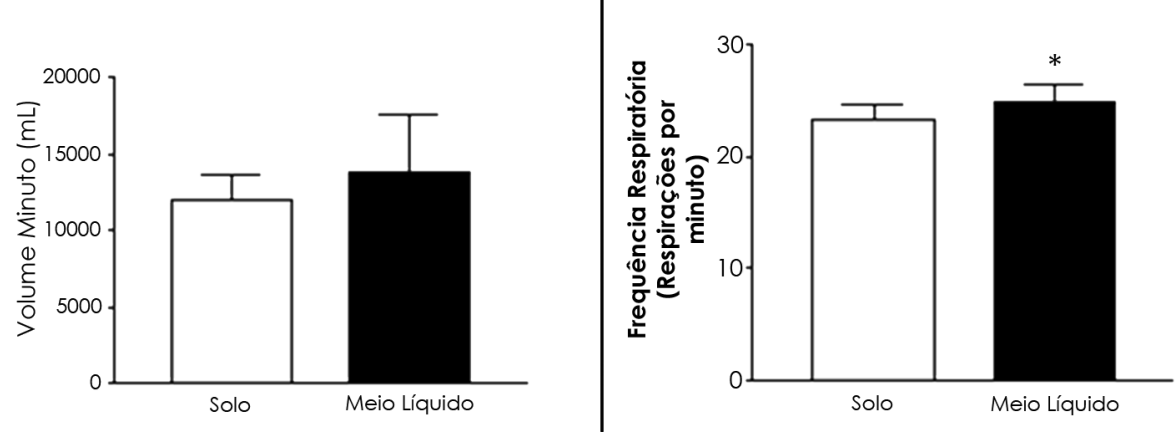

Figura 2. Dados de VM (A), VC (B) e FR (C) de pacientes com DMD em solo e meio líquido. * vs. Meio Líquido (Wilcoxon, $p<0,05$ ); N: 15

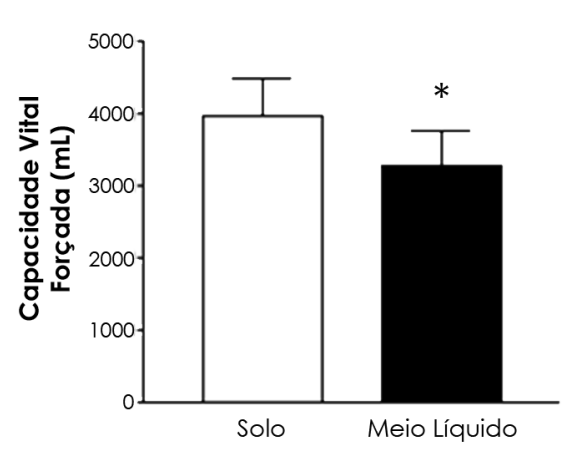

A

Figura 3. Dados de CVF (A) e correlação dos valores de CVF (B) de pacientes com DMD em solo e meio líquido. * vs. Meio Líquido (A: Wilcoxon e B: Spearman, $p<0,05)$; N: 15

O valor de PFE apresentou-se estatisticamente menor no meio líquido quando comparados com os dados do solo $(p=0,024)$, e esses valores quando correlacionados apresentaram correlação positiva entre os dados obtidos em solo e em meio líquido (Figura 4).

\section{DISCUSSÃO}

De acordo com os resultados obtidos neste estudo, observa-se que a $\mathrm{SpO}_{2}$ sofreu uma redução estatisticamente significante após a avaliação no meio líquido. Este resultado está de acordo com Cole \& Becker, ${ }^{13}$ quando relataram que a capacidade de difusão está levemente diminuída quando indivíduo está imerso no nível de C7. Em virtude da base dos pulmões estarem menos ventiladas e perfundidas pelo deslocamento de sangue das extremidades para o ápice do tórax, há um ingurgitamento dos vasos pulmonares, diminuição na capacidade de difusão e consequentemente déficit nas trocas gasosas.

Arborelius e colaboradores relatam que durante a imersão a FC pode permanecer inalterada ou ter uma redução reflexa. ${ }^{18}$ Este dado corrobora os resultados do presente estudo, na qual não foi observada diferença nesta variável quando comparada entre os meios avaliados.

Não foram encontradas alterações entre os valores de PI Máx. e PE Máx. quando analisados em solo e em meio líquido. Estes resultados sugerem que a pressão hidrostática exercida sobre a caixa torácica no nível de imersão analisado não seja suficiente para impor uma resistência na musculatura respiratória ao ponto de reduzir a capacidade de contração destes músculos. A pressão hidrostática aumenta com o aumento da profundidade. Tendo em vista que os pacientes aqui avaliados estavam sentados com imersão no nível de $\mathrm{C7}$, a pressão hidrostática exercida na caixa torácica é menor, próxima de $20 \mathrm{~cm} \mathrm{H} \mathrm{O}^{19}$ pois este segmento está mais próximo da superfície da água, logo, menos influenciada por ela.

Quando o indivíduo estava em imersão, o VC não sofreu alteração, porém, houve aumento da FR e consequentemente aumento significativo do VM. Chouckroun et al., ${ }^{17}$ avaliando indivíduos saudáveis em diferentes temperaturas, concluíram que em água termoneutra $\left(34^{\circ} \mathrm{C}\right)$ o VC não sofria alteração. Embora a temperatura da água não tenha influenciado na alteração do VC, outras variáveis são temperatura-dependentes. Segundo Bach, no estágio inicial do comprometimento pulmonar, o VC permanece inalterado. ${ }^{20}$

A FR sofreu um aumento quando pacientes estavam imersos em decorrência da redução da expansibilidade da caixa torácica e da complacência pulmonar. Desta forma, 


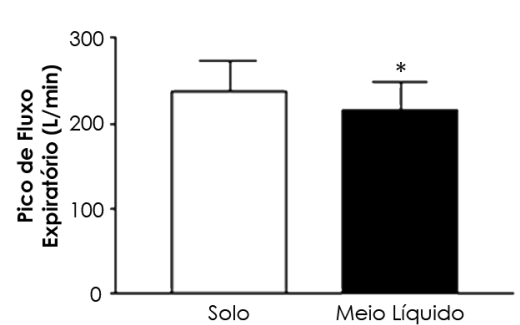

\section{A}

Figura 4. Dados de PFE (A) e correlação dos valores de PFE (B) de pacientes com DMD em solo e meio líquido. * vs. Meio Líquido (A: Wilcoxon e B: Spearman, $p<0,05)$; N: 15

o indivíduo precisa respirar mais vezes por minuto para suprir suas demandas. ${ }^{13}$ Sob estas condições, ocorrem alterações circulatórias, respiratórias e biomecânicas, o que leva a uma sobrecarga do sistema pulmonar e a um aumento de $60 \%$ no trabalho respiratório, justificando estes resultados.

Quanto à CVF, foi observada uma redução de $17 \%$ quando os pacientes estavam imersos. Em indivíduos normais esta redução é de aproximadamente $10 \% \cdot{ }^{21-25} \mathrm{~A}$ literatura enfatiza que, durante a imersão cerca de $700 \mathrm{ml}$ de sangue são deslocados para a área do tórax, sendo que $200 \mathrm{ml}$ vão para o coração e $500 \mathrm{ml}$ vão para região intratorácica. ${ }^{18,21,22}$

Para estes autores, a redução da CV ocorre pelo aumento deste volume sanguíneo intratorácico, levando a uma diminuição na complacência pulmonar.

PFE apresentou uma redução quando indivíduo estava em imersão. Segundo Cole \& Becker, esta diminuição é decorrente do aumento da pressão na caixa torácica, diminuindo a sua circunferência e, consequentemente, aumentando do fluxo aéreo nas vias aéreas. ${ }^{13}$ Estudos sobre biofisiologia da imersão em indivíduos normais também apóiam esta relação. ${ }^{22}$

Existe um limite mínimo de valor de CVF e PFE para previsão de sobrevida, de acordo com o último consenso a respeito de DMD, para que estes pacientes tenham indicação para métodos de assistência à tosse. ${ }^{2}$ Os valores citados neste texto são: CVF $<1 \mathrm{~L}$ e PFE $<270 \mathrm{~L} / \mathrm{min}$. Os resultados obtidos neste trabalho apontam para uma redução destas variáveis em meio líquido, sendo que há correlações positivas entre os valores em solo e meio líquido. De acordo com essas informações, pode-se sugerir um cálculo piloto para predizer valores de CVF e de PFE no meio líquido a partir de valores quantificados em solo. Apesar do número de indivíduos avaliados não ser expressivo, pôde-se chegar a uma correlação entre os valores da CVF e PFE entre o solo e o meio líquido, resultando em uma reta com pouca dispersão entre os dados. Portanto, de acordo com essa regressão, é possível chegar às seguintes equações:

$$
\begin{aligned}
\mathrm{CVF}_{\text {liq }} & =0,6188^{*} \mathrm{CVF}_{\text {solo }}+827,40 \\
\mathrm{PFE}_{\text {liq }} & =0,8354 * \mathrm{PFE}_{\text {solo }}+16,84
\end{aligned}
$$

$\mathrm{CVF}_{\text {liq }}$ e $\mathrm{CVF}_{\text {solo }}$ representam valores de CVF em meio líquido e em solo, respectivamente, e $\mathrm{PFE}_{\text {liq }}$ e $\mathrm{PFE}_{\text {solo }}$ representam valores de PFE em meio líquido e em solo, respectivamente. Não foi encontrada literatura similar aos dados do presente trabalho, porém, acredita-se que ao elevar o número de pacientes incluídos nesta correlação, é possível predizer com maior precisão a redução da CVF e do PFE causada pela imersão em pacientes com DMD.

\section{CONCLUSÃO}

Durante a imersão, no nível de C7, de pacientes com distrofia muscular de $\mathrm{Du}$ chenne, foi possível observar aumento da FR e diminuição da $\mathrm{SpO}_{2}$, CVF e PFE. Tendo em vista as alterações respiratórias comumente encontradas nestes pacientes, tais resultados ressaltam a importância da avaliação dos parâmetros ventilatórios antes da utilização da Fisioterapia Aquática na reabilitação.

Levando-se em consideração os dados que correlacionam os valores de CVF e PFE em solo e em meio líquido, sugere-se uma forma de estimar estes parâmetros ventilatórios em meio líquido a partir dos dados quantificados em solo. Ainda que estes valores apurados em solo estejam superiores aos indicados pelo Consenso e de acordo com as estimativas para o meio líquido, estiverem abaixo do nível predito, dever-se-ia avaliar com maior cautela a indicação para realização de fisioterapia aquática.

\section{REFERÊNCIAS}

1. Silva JDM, Costa KS, Cruz MC. Distrofia muscular de Duchenne: um enfoque cinesioterapêutico. Lato \& Sensu. 2003;4(1):3-5.

2. Finder JD, Birnkrant D, Carl J, Farber HJ, Gozal D, lannaccone ST, et al. Respiratory care of the patient with Duchenne muscular dystrophy: ATS consensus statement. Am J Respir Crit Care Med. 2004;170(4):456-65.

3. Slutzky, LC. Fisioterapia respiratória nas doenças neuromusculares. Rio de Janeiro: Revinter; 1997.

4. Inkley SR, Oldenburg FC, Vignos PJ Jr. Pulmonary function in Duchenne muscular dystrophy related to stage of disease. Am J Med. 1974;56(3):297-306.

5. McDonald CM, Abresch RT, Carter GT, Fowler WM Jr, Johnson ER, Kilmer DD, et al. Profiles of neuromuscular diseases. Duchenne muscular dystrophy. Am J Phys Med Rehabil. 1995;74(5 Suppl):S70-92.

6. Hapke EJ, Meek JC, Jacobs J. Pulmonary function in progressive muscular dystrophy. Chest. 1972;61(1):41-7.

7. Rideau Y, Jankowski LW, Grellet J. Respiratory function in the muscular dystrophies. Muscle Nerve. 1981;4(2):155-64.

8. Bäckman E, Nylander E. The heart in Duchenne muscular dystrophy: a non-invasive longitudinal study. Eur Heart J. 1992;13(9):1239-44.

9. Adams MA, Chandler LS. Effects of physical therapy program on vital capacity of patients with muscular dystrophy. Phys Ther. 1974;54(5):494-6.

10. Barbé F, Quera-Salva MA, McCann C, Gajdos P, Raphael JC, Lattre J, et al. Sleep-related respiratory disturbances in patients with Duchenne muscular dystrophy. Eur Respir J. 1994;7(8):1403-8.

11. Griggs RC, Donohoe KM, Utell MJ, Goldblatt D, Moxley RT 3rd. Evaluation of pulmonary function in neuromuscular disease. Arch Neurol. 1981;38(1):9-12.

12. Hahn A, Bach JR, Delaubier A, Renardel-Irani A, Guillou C, Rideau Y. Clinical implications of maximal respiratory pressure determinations for individuals with Duchenne muscular dystrophy. Arch Phys Med Rehabil. 1997;78(1):1-6.

13. Cole AJ, Becker BE. Comprehensive aquatic therapy. Philadelphia: Elsevier; 2004.

14. Taylor NA, Morrison JB. Lung volume changes in response to altered breathing gas pressure during upright immersion. Eur J Appl Physiol Occup Physiol. 1991;62(2):122-9.

15. Taylor NA, Morrison JB. Static and dynamic pulmonary compliance during upright immersion. Acta Physiol Scand. 1993;149(4):413-7.

16. Taylor NA, Morrison JB. Static respiratory muscle work during immersion with positive and negative respiratory loading. J Appl Physiol. 1999;87(4):1397-403.

17. Choukroun ML, Kays $C$, Varène $P$. Effects of water temperature on pulmonary volumes in immersed human subjects. Respir Physiol. 1989;75(3):255-65.

18. Arborelius M Jr, Ballidin UI, Lilja B, Lundgren CE. Hemodynamic changes in man during immersion with the head above water. Aerosp Med. 1972;43(6):592-8. 
19. Hall J, Bisson D, O'Hare P. The physiology of immersion. Physiology. 1990;76:517-21.

20. Bach JR, Gonçalves MR. Noninvasive ventilation or paradigm paralysis? Eur Respir J. 2004;23(4):651.

21. Agostoni E, Gurtner G, Torri G, Rahn H. Respiratory mechanics during submersion and negativepressure breathing. J Appl Physiol. 1966;21(1):251-8
22. Hong SK, Cerretelli P, Cruz JC, Rahn H. Mechanics of respiration during submersion in water. J Appl Physiol. 1969;27(4):535-8.

23. Hong SK, Ting EY, Rahn H. Lung volumes at different depths of submersion. J Appl Physiol. 1960;15:550-3.
24. Craig $A B \mathrm{Jr}$, Ware DE. Effect of immersion in water on vital capacity and residual volume of the lungs. J Appl Physiol. 1967;23(4):423-5.

25. Carey CR, Schaefer KE, Alvis HJ. Effect of skin diving on lung volumes. J Appl Physiol. 1956;8(5):519-23. 